\title{
Golden Rice - five years on the road - five years to go?
}

\author{
Salim Al-Babili and Peter Beyer
}

University of Freiburg, Center for Applied Biosciences, Schänzlestr. 1, 79104 Freiburg, Germany

\begin{abstract}
Provitamin A accumulates in the grain of Golden Rice as a result of genetic transformation. In developing countries, where vitamin A deficiency prevails, grain from Golden Rice is expected to provide this important micronutrient sustainably through agriculture. Since its original production, the prototype Golden Rice has undergone intense research to increase the provitamin A content, to establish the scientific basis for its carotenoid complement, and to better comply with regulatory requirements. Today, the current focus is on how to get Golden Rice effectively into the hands of farmers, which is a novel avenue for public sector research, carried out with the aid of international research consortia. Additional new research is underway to further increase the nutritional value of Golden Rice.
\end{abstract}

\section{Golden Rice - intent and role}

Golden Rice (Oryza sativa, GR) is the generic name given to genetically modified rice that produces $\beta$-carotene (provitamin A) in the endosperm. This name is derived from the yellow color of the grain that is visible after milling and polishing, the procedure that is routinely employed to remove the outer grain layers. The research that led to the development of GR was initiated to help alleviate vitamin A deficiency (VAD), which represents a major global health problem (Box 1). Through agriculture and local trade, GR is expected to reach the target populations, namely the urban poor and rural populations, particularly those living in remote areas. Here GR is expected to complement more traditional interventions, such as industrial food fortification and supplementation, effectively and sustainably. These interventions rely on centrally processed food items, on the maintenance of adequate distribution logistics and on the specific targeting of deficient populations, and require significant on-going costs to be sustained. GR, in principle, should require little more than the costs of reliable seed production systems for its continued deployment.

Genetic engineering was the only way to produce GR because there is no rice germplasm capable of synthesizing carotenoids in the endosperm available. The transgenic approach has become feasible during recent years for two reasons: (i) the rapid progress in the development of rice transformation technologies through

Corresponding author: Beyer, P. (peter.beyer@biologie.uni-freiburg.de). biolistic methods as well as using Agrobacterium; (ii) the availability of the almost complete molecular elucidation of the carotenoid biosynthetic pathway in numerous bacteria and plants, which provides ample choice of bacterial genes and plant cDNAs to select from. In this review we consider the development of GR since its inception five years ago and take our bearings on progress and on the plans to deliver the product into the hands of farmers and consumers.

\section{Development and improvements to date}

An experimental Japonica rice line (Taipei 309) was used to produce the prototypes of GR [1] by Agrobacteriummediated transformation. The rationale for the genes used in the DNA constructs (Figure 1; pB19hpc and pZPsC) was based on a series of pre-experiments that showed that wild-type endosperm contained the precursor molecule geranylgeranyl diphosphate, which could be used upon transformation by the enzyme phytoene synthase from daffodil, yielding the uncolored carotene phytoene [2] (Figure 2). No colored carotenoids, products of the metabolism of phytoene, could be measured. This led to the inference that no further carotenogenic enzymes or, at least not phytoene desaturase, were functionally expressed. Therefore, further complementation was needed and achieved by introducing the bacterial carotene desaturase CRTI along with PSY (pB19hcp, Figure 1). CRTI substitutes for the two plant carotene desaturases, phytoene desaturase and $\zeta$-carotene desaturase, as well as for the carotene-isomerase (CRTISO), and converts phytoene into lycopene directly (Figure 2 ). The cDNA for lycopene- $\beta$ cyclase ( $\beta$-LCY), which is necessary to complete the pathway that leads towards $\beta$-carotene formation, was introduced by co-transformation (pZLcyH, Figure 1) so that transgenic rice plants were recovered that carried two transgenes coding for PSY and CRTI, or three coding for PSY, CRTI and $\beta$-LCY. These prototypes produced $\beta$-carotene regardless of the presence or absence of $\beta$-LCY (see below). The GR prototypes contained up to $1.6 \mu \mathrm{g} / \mathrm{g}$ of carotenoids with an event-dependent-proportion of provitamin A carotenoids (the integration of foreign DNA into a genome is referred to as 'event') and contained the aph IV-gene as the selectable marker.

The prototypes demonstrated the feasibility of GR but more rigorous criteria needed to be met for product development towards deregulation (Box 2), including increasing the content of provitamin A carotenoids, avoiding the antibiotic selectable marker and optimizing 


\section{Box 1. Vitamin A deficiency}

Vitamin A comprises a family of retinoids. Retinol, the best-known retinoid, is common in food derived from animals such as liver, eggs and fish-products. Food derived from plant sources also contributes to our Vitamin A supply by providing provitamin A carotenoids, for example, $\beta$-carotene. The ratio of vitamin $A$ derived from a plant diet differs significantly depending on the economic wealth. For instance, only $26-34 \%$ of vitamin A consumed by Americans is provided by provitamin A carotenoids (http://ods.od. nih.gov/factsheets/cc/vita.html). By contrast, in many developing countries, provitamin A carotenoids have a much larger significance, for example, in rural Bangladesh, only $3 \%$ of the calorie intake is from animal sources but nearly $80 \%$ comes from rice [31]. Prolonged predominant consumption of such staples lacking provitamin A carotenoids contributes to vitamin A deficiency (VAD).

Vitamin $A$ is essential for vision, immune response, epithelial cell growth and repair, bone growth, reproduction, maintenance of the surface linings of the eyes and the epithelial integrity of respiratory, urinary and intestinal tracts. Vitamin A is also important for embryonic development and regulation of adult genes.

VAD leads to various disorders and increased disease susceptibility. One of the earliest symptoms of VAD is night blindness. Prolonged deficiency results in drying of the conjunctiva. With continued vitamin A deficiency, the drying extends to the cornea (xerophthalmia).
The cornea eventually shrivels and becomes ulcerated (keratinomalacia). Finally, inflammation and infection occur in the interior of the eye, resulting in total and irreversible blindness. In addition to causing eye diseases, VAD increases the severity of measles and diarrhea, leading to increased child mortality. This increased infectious morbidity and mortality are apparent even before the appearance of xerophthalmia. VAD can result in subclinical disorders such as impaired iron mobilization, disturbed cellular differentiation and depressed immune response [32]. The severity of this malnutrition problem is evident from studies conducted by the World Health Organization (WHO) showing that correcting VAD can reduce child mortality by $\sim 23 \%$ (http://www.who.int/vaccines-diseases/en/vitamina/science/sci01.shtml).

According to the WHO, VAD represents a public health problem in more than 118 countries and affects between 140 million and 250 million preschool children worldwide. It is estimated that between 250000 and 500000 vitamin A-deficient children become blind every year, half of them die within 12 months of loosing their sight. VAD is the primary cause of childhood blindness worldwide and is now recognized as a major contributing factor in an estimated 1 million 3 million child deaths each year. In addition, nearly 600000 women die from childbirth-related causes each year, the vast majority of them from complications that could be reduced through better nutrition, including provision of vitamin A.

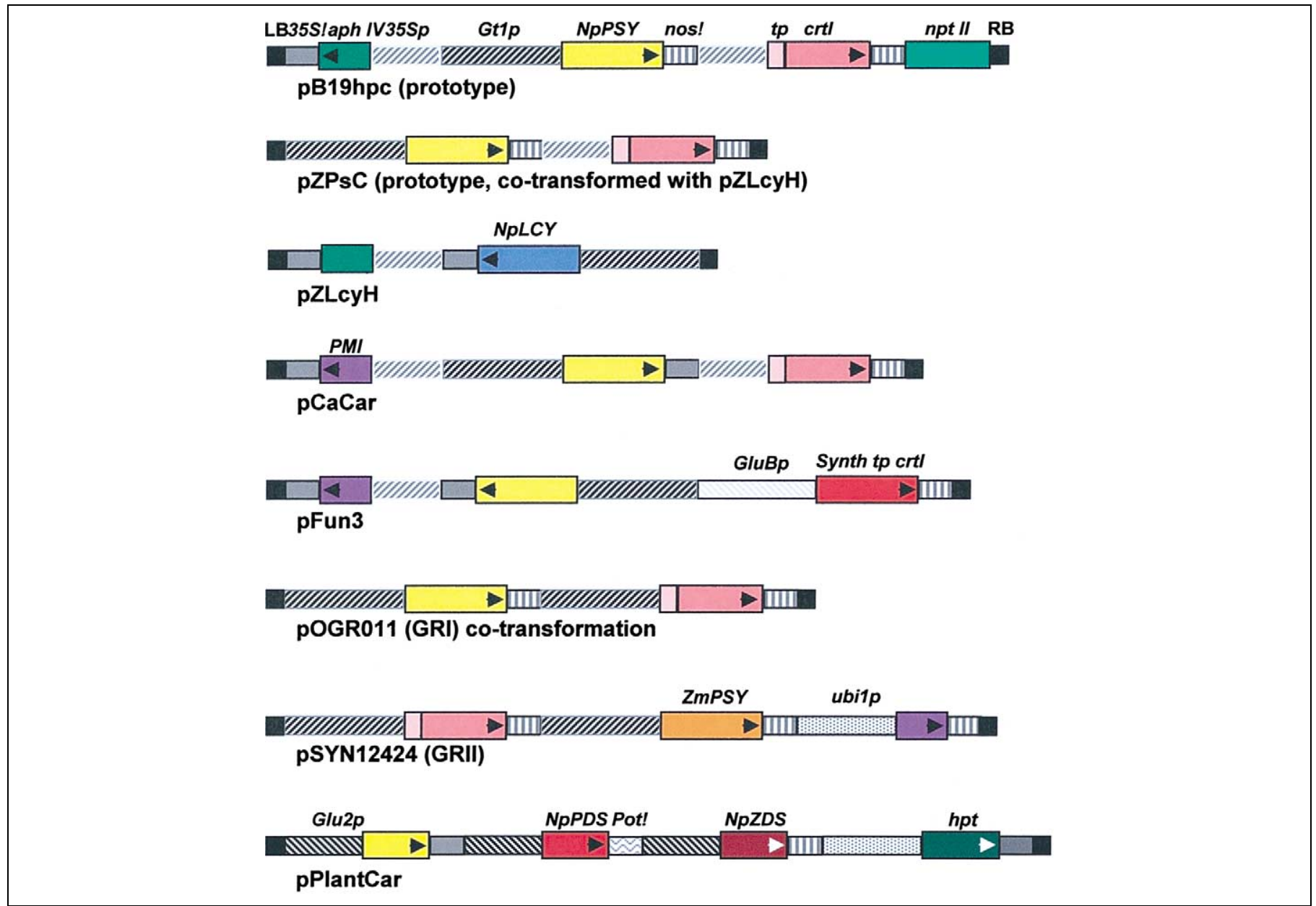

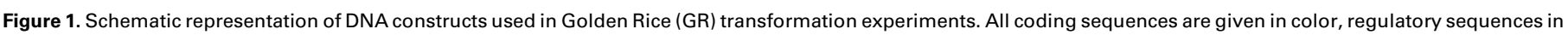
gray. Colors and patterns are codes that are maintained in all diagrams. Abbreviations: aph, aminoglycoside-phosphotransferase; crtl, carotene-desaturase from Erwinia

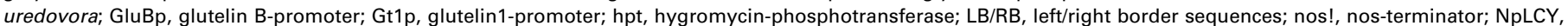

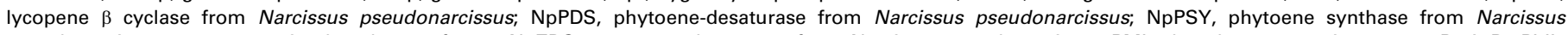
pseudonarcissus; npt, neomycin-phosphotransferase; NpZDS, ל-carotene-desaturase from Narcissus pseudonarcissus; PMI, phosphomannose-isomerase; Pot!, PotPI-II-

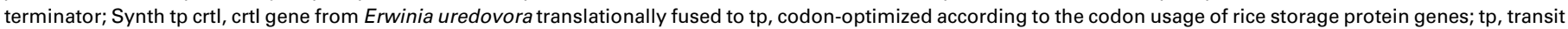

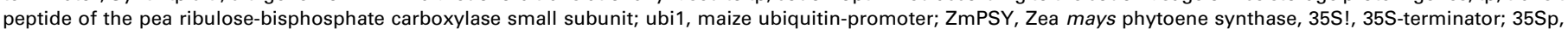
CaMV 35S-promoter. 


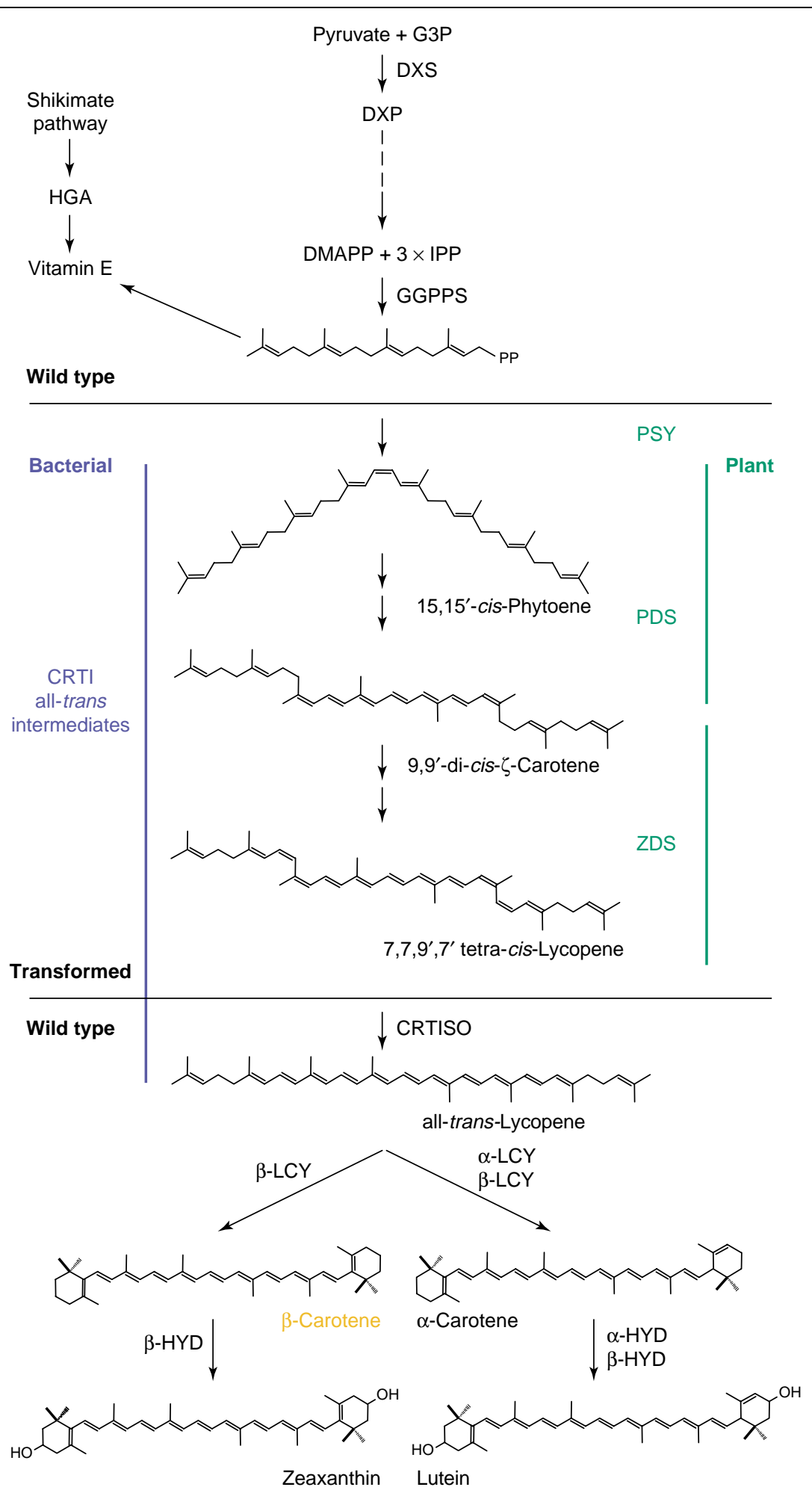

$\overline{\text { TRENDS in Plant Science }}$

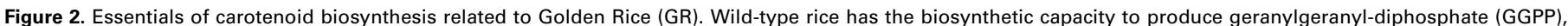

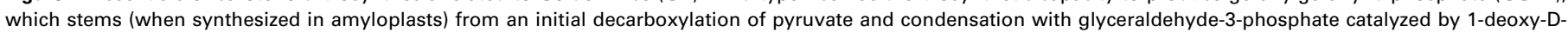

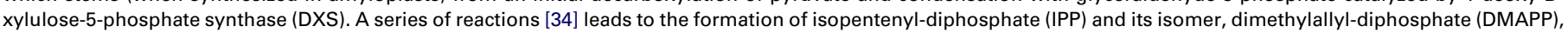

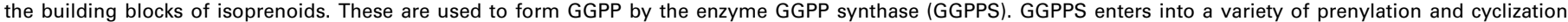

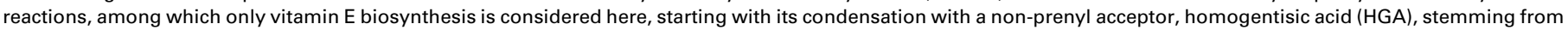

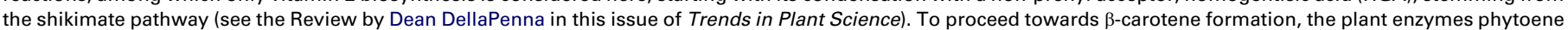

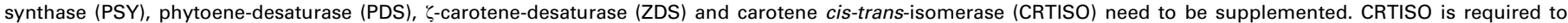

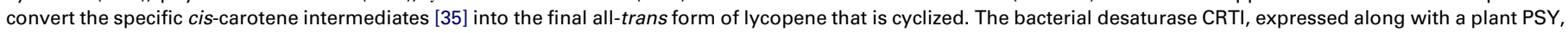

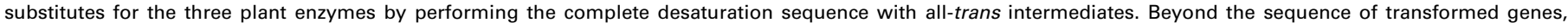

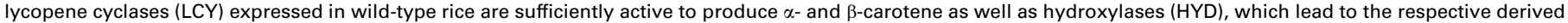
hydroxylated xanthophylls. The intrinsic activity of CRTISO is required in experimental GR versions expressing the plant desaturases. 


\section{Box 2. Deregulation}

Deregulation is one term used for the process that eventually leads to the registration of a product and the granting of its unlimited commercial or non-commercial use. The national authorities of the respective countries in which the product is intended for use govern deregulation of GMO plants and the foods derived from these. The process is driven by a science-based risk assessment of the intended product and the environments into which it will be released. Current data requirements can be extensive and are comprehensive, and require the submission of publication quality data reports. The development of these materials can be timeconsuming and costly. The studies required to compile a regulatory dossier fall in two categories: event-dependent and eventindependent studies.

Event-independent studies can include biochemical analysis (function, specificity and mode of action), studies on toxicity and the allergenic potential of the newly expressed proteins. In addition, dietary exposure modeling and bioavailability studies can be conducted with the newly expressed proteins and, in the case of enzymes, with the products produced by catalysis.

Event-dependent studies vary by country and can encompass the characterization of the quality of integration, such as copy number, the determination of the site of integration in the host genome, demonstration of the absence of gene disruption, completeness of integrated DNA sequences, and presence of the marker gene at the same locus and absence of vector DNA sequences. Phenotypic and biochemical evidence for trait stability, including the monitoring of gene expression levels at key growth stages and Mendelian inheritance over several generations, are usually components of these studies. Field performance of typical agronomic traits, such as yield, and pest and disease resistance, is an additional issue.

the choice of genes. Moreover, it needed to be shown that the technology functioned equally well in agronomically important rice genotypes for those areas with $\mathrm{VAD}$, for example, Indica rice varieties. In addition, questions had to be answered on the effectiveness of the provitamin A accumulated in grains to combat VAD. This is governed by two parameters (besides those related to public acceptance): the amount of provitamin A in the grain and its bioavailability. Several steps have been taken to meet these criteria, both in the public sector as well as by the private sector partner, Syngenta (http://www.Syngenta. com; Box 3).

To produce GR suitable for deregulation, several transformations were performed, continuing with Agrobacteria because of the more defined transgene integration pattern that this technique usually produces. In the public sector, these transformation events were conducted using the vector pCaCar (Figure 1), which relied on the phosphomannose-isomerase (Positech ${ }^{\circledR}$ ) sugar-based selection system [3] as a replacement for the antibiotic resistance gene used in the prototypes. These transformation experiments showed that the principle of GR was applicable to Indica varieties, such as IR64 [4,5]. In parallel, Syngenta scientists produced many transformation events using the Javanica cultivar Cocodrie with a modified vector in which CRTI was under endosperm-specific promoter control (Figure 1, pOGR011). By contrast, in public sector experiments, this gene was controlled by the CaMV $35 \mathrm{~S}$ promoter (pCaCar). The transgenic plants produced by Syngenta scientists were ultimately devoid of a selectable marker gene because of the co-transformation technique
The assessment is also based on the comparison of the compositional analyses from materials harvested at different locations with the equivalent non-transgenic crop (and food) comparator. Event-dependent regulatory data are usually not gathered until the developer is satisfied with the technical performance of the product and a few events (usually one) have been chosen. Assessment of the potential risks of $\mathrm{GM}$ crops is on a case-by-case basis within a scientific framework. A GMO crop producing $\beta$-carotene must be viewed differently than a crop that produces an insecticide. Similarly, GR events expressing a phytoene synthase from maize, a widely consumed crop, might be viewed differently than one using the enzyme from a non-food plant, such as daffodil.

From a scientific point of view it is often hard to understand why randomly mutagenized crop plants can enter into breeding lines easily, whereas GMOs, with considerably fewer genetic modifications, have a much more rigorous assessment. To have rational, sciencebased regulatory requirements it would be necessary to realize the benefits (not only potential risks) that GMO crops can provide (see lecture by Ingo Potrykus at http://www.syngentafoundation.com/ golden_rice/index.htm). It is unhelpful to raise the regulatory requirements to the point where anything that appears technically feasible is being requested or is being offered to be applied [33], with the only justification that the genetically modified organism involved is little understood. This strategy can raise costs to unaffordable levels. We do not understand bred varieties with their complex genomic changes much better at the molecular level, but we tend to consider the traditional way of producing new varieties safe in contrast to the novel way of producing GMOs even though this is not based on any rational evaluation. It is the final product and not the technology used to produce it that should be scrutinized.

employed, thus allowing the removal of the marker gene through selfing when integrated into different sites in the genome. The finally chosen events are known as GR1 in the Golden Rice Network (Box 3). They represent the bestinvestigated GR events available to date that have undergone intense molecular characterization and evaluation, including the first field trial in 2004. These GR1 events showed significantly higher carotenoid contents, up to $6.0 \mu \mathrm{g} / \mathrm{g}$, whereas the public sector events showed a maximum of $1.6 \mu \mathrm{g} / \mathrm{g}$. Explanations for this difference might lie in the use of a different promoter, the different biochemical background of the cultivar used, or positional effects and, thus, in the larger number of independent transformation events produced. Subsequent introgression into different cultivars of rice, which is currently underway, is expected to shed light on some of these questions.

The amount of carotenoids formed in the prototype GR $(1.6 \mu \mathrm{g} / \mathrm{g})$ has been severely criticized, particularly in the media and by opponents of the technology, even though a socio-economic ex ante impact analysis (an estimation of expected effects) carried out in the Philippines resulted in a positive judgment on GR [6] (additional ex ante studies are underway in Bangladesh and India based on the now improved provitamin A content in GR1 and GR2, see below). Nevertheless, the past 3-4 years have been dominated by experiments aimed at increasing the provitamin A production in rice endosperm. Insufficient knowledge of the rate-limiting steps in the provitamin A pathway in rice endosperm led to three different approaches being considered. One is to assume that the amount of precursor molecules of geranylgeranyl- 


\section{Box 3. The Partnership}

The product development of Golden Rice (GR) is based on a 'deal', secured by licenses, signed between the inventors and Syngenta, in which the inventors granted exclusive worldwide commercial rights to the invention. In exchange, Syngenta granted a humanitarian license together with all relevant associated intellectual property necessary to attain 'Freedom-to-Operate' in developing countries. (Today, Syngenta still retains the commercial rights but is not interested in executing them; see press release in $2004 \mathrm{http}: / / \mathrm{www}$.syngenta.com/ en/media/intro.aspx). Syngenta further agreed to support the inventors in their task to introduce the technology free of charge to nutritionally deprived farmers and their customers in developing countries. This resulted in the creation of a private-public partnership between the inventors and Syngenta. This partnership facilitated the licensed access to a small number of what were then key technologies, with relevant intellectual property held by several players in the field of biotechnology. These included Bayer (http://www.bayer.com), Monsanto (http://www.monsanto.com), Novartis (http://www.novartis. com), and Mogen (formerly), as well as Syngenta. In addition, Syngenta scientists during the past years have contributed substantially to the improvement of the technology [8] as well as to product development and regulatory expertise and advice.

diphosphate (GGPP) is limiting. Here, the enzyme 1-deoxy-D-xylulose-5-phosphate synthase (DXS) could be the key enzyme because of its ability to link the primary C3 metabolism to isoprenoid biosynthesis and its postulated function in regulating carotenoid biosynthesis [7]. The other two avenues considered were how to optimize the introduced enzymes PSY and CRTI to increase their expression level or enzymatic efficiency.

Considering how to increase the expression of CRTI seemed reasonable because its low expression in the prototype events rendered it undetectable in western blots. This was significantly improved by using the vector pFun3 (Figure 1), carrying a codon-optimized version of CRTI under endosperm-specific promoter control. However, the carotenoid amount was not significantly increased (S. Al-Babili et al., unpublished). Similarly, the introduction of DXS from daffodil in combination with pFun3 using a co-transformation approach did not result in the desired improvement. Thus, neither the flux of carbon into prenyllipid synthesis nor the efficiency of CRTI is the rate-limiting step in carotenoid synthesis in GR.

This is corroborated by results obtained by Syngenta scientists who focused on optimizing the expression of PSY [8]. A side-by-side comparison of PSYs from different plants was carried out in a maize tissue culture model system, which revealed substantial differences in carotenoid accumulation. Moreover, those stemming from known carotenoid overproducers, such as from daffodil (used in all previous versions of GR), carrot and tomato, were less effective, whereas there was a marked increase in carotenoid biosynthesis with PSYs from Graminaceae (i.e. rice itself or maize). These findings translated into large increases in the amounts of carotenoids synthesized, up to $37 \mu \mathrm{g} / \mathrm{g}$ (of which $84 \%$ is $\beta$-carotene), which led to the development of a new version of Golden Rice (GR2; Figure 3) that relies on the maize PSY enzyme (pSYN12424, Figure 1). This demonstrates that phytoene synthesis is a rate-limiting step in rice and also helps to explain why the experiments to optimize CRTI transgene
The humanitarian GR license allows the use of the pro-Vitamin A technology free of cost for farmers with a farming income of up-to US $\$ 10000 /$ year and permits national, but not international, trade. The license obliges licensees, which are all public sector research institutions, to use and to develop the technology in accordance with the respective national and international regulations. To date, 16 research institutions (in India, The Philippines, China, Bangladesh, Indonesia, Vietnam and South Africa) are licensees and contribute to GR product development as a consortium, termed the Golden Rice Network. Most of this research is funded by national grants from the countries in which the research institutions are based, by the Rockefeller Foundation (http://www.rockfound.org), USAID (http://www.usaid.gov), and the HarvestPlus Consortium (http://www. harvestplus.org). Syngenta's in-house work for the project has also been a significant contribution.

The Humanitarian Board, an advisory panel, has been formed by several individuals with complementary and relevant expertise from public sector institutions and Syngenta, with the mission to ensure the intended use of the technology and to facilitate and to steer the overall program (for additional information see http://www. goldenrice.org).

expression did not yield the desired effect on their own. Similarly, there seems to be no shortage of GGPP as a precursor, which might enable further increases in provitamin A content, if required. Thus, the situation seems somewhat similar to the one found with transgenic canola, which relied on the overexpression of a bacterial PSY to increase carotenoid content markedly [9]. However, the overall biochemistry might not be comparable because the rice grain is not an oil-rich seed and the need for additional desaturase activity has been demonstrated in rice.

$\beta$-Carotene is also known as provitamin A because after absorption it undergoes central enzymatic cleavage by a recently identified oxygenase [10] to yield vitamin A.

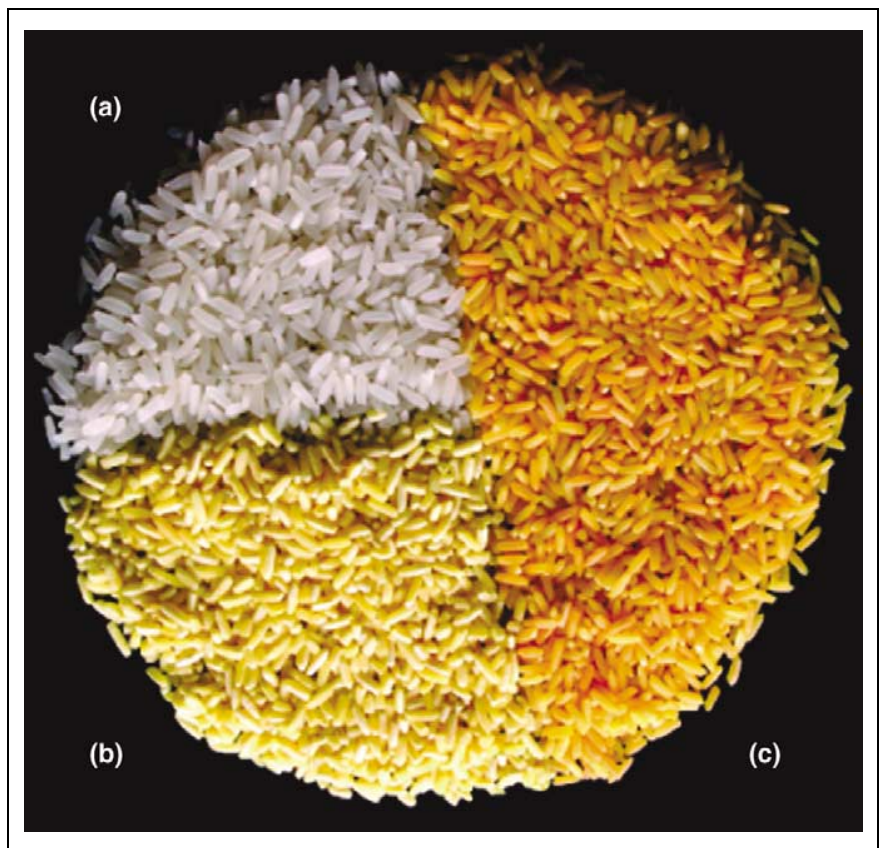

Figure 3. Golden Rice colors. (a) Wild-tape rice; (b) GR1, expressing the phytoene synthase from daffodil along with CRT1; (c) GR2 expressing the phytoene synthase from maize along with CRTI. Photograph courtesy of Aron Silverstone. 
The efficiency of absorption and enzymatic cleavage governs bioavailability and bioefficacy, respectively [11]. The US National Academy of Sciences Institute of Medicine developed conversion factors to address this overall process when it revised the recommended daily allowance (RDA) for vitamin A [12]. The conversion factor for $\beta$-carotene from a mixed diet is 12 . Using this conversion factor and a conservative estimation of only $24 \mu \mathrm{g} / \mathrm{g}$ of provitamin A, $72 \mathrm{~g}$ of dry GR2 polished rice would provide $50 \%$ of the RDA for children [8]. It is therefore expected that GR2 will be able to contribute to alleviating VAD. However, the precise determination of the conversion factor to be applied for the rice endosperm source and in different food matrices still needs to be determined. A human feeding trial is planned to take place in early 2006 that should help to answer this question.

\section{Carotenoid biosynthesis in the rice endosperm}

A scientific question that has accompanied all versions of GR has been to explain how $\beta$-carotene and xanthophylls are formed even though neither lycopene cyclase nor a carotene hydroxylase [13] are transformed (i.e. the biochemical pathway proceeds beyond the end-point expected by the enzymatic activity of the expressed transgenes). Published evidence pointed to the occurrence of a feedback regulatory phenomenon in transgenic tomato [14] capable of markedly modifying the expression of several endogenous carotenoid biosynthetic genes. The bacterial CRTI transgene in particular was suspected to be the cause of this phenomenon because this desaturase differs from the plant-type desaturases, not only structurally but also in the nature of the lycopene produced: all-trans in the bacterial system but specifically poly-cis when produced by plant-type carotene desaturases (Figure 2) [15]. In plants, this requires an additional enzyme, the recently identified carotene cis-trans-isomerase (CRTISO) [16,17], to enable cyclization and the production of all-trans $\beta$-carotene. The artificial accumulation of all-trans lycopene in daffodil flowers by the application of the cyclase inhibitor CPTA [2-( $p$-chlorophenylthio)triethylammonium chloride] led to altered expression levels of carotenogenic mRNAs and seemingly underscored the relevance of such a feedback mechanism [18]. However, the use of Real-Time PCR to quantify the levels of rice-encoded carotenogenic mRNAs in the endosperm showed no indication of such a regulatory principle. Wild-type rice endosperm was shown to express mRNAs for all the relevant carotenoid biosynthetic genes, with the exception of PSY, which was effectively absent [19]. The occurrence of $\beta$-LCY-mRNA explains why it is not needed as a transgene and the hydroxylase transcripts explain the formation of xanthophylls in GR. However, the presence of the phytoene desaturase (PDS) and $\zeta$-carotene desaturase (ZDS) transcripts conflicts with earlier data that showed that expression of daffodil PSY as the sole transgene in rice endosperm yielded only phytoene but not colored carotenoids. However, the detection of PDS and ZDS mRNAs does not necessarily imply the presence of sufficient functional protein. Supporting this suggestion, the overexpression of PDS and ZDS along with the daffodil
PSY (Figure 1, pPlantCar) resulted in $\beta$-carotene and xanthophyll accumulation [19]. This suggests that the cofactor requirements of PDS, including the presence of active redox pathways [20], are met in the endosperm. The introduction of the plant carotene desaturation pathway by transformation also demonstrated the existence of endogenous CRTISO activity because all-trans $\beta$-carotene was formed [19]. Because of the relevance of PSY for the reaction velocity of the overall pathway, the relative effectiveness of the two desaturation systems cannot be estimated to date.

It is interesting to note that although no statistically significant alterations in specific mRNA levels were detected upon constitutive expression of CRTI, a decrease in lutein in favor of $\beta$-carotene-derived xanthophylls was observed in leaves of rice and Arabidopsis [19]. To avoid such effects that might influence the photosynthetic performance, the preferred option is to express CRTI under endosperm-specific promoter control, under which conditions this effect does not occur.

\section{Open scientific questions}

It is worthwhile, both in terms of enzymology and for future biotech application of GR technologies, to strive to understand the molecular basis of the large differences in effectiveness of PSY orthologs. Given the high degree of homology between plant PSYs, subtle but effective differences in primary structure are expected to govern the adaptation of the enzyme to its biochemical surroundings. Perhaps not surprisingly, the two best-performing PSY proteins (from rice and maize) were more similar to each other than to any of the other PSYs used [8]. Adaptations can be related to specific biochemical microenvironments affecting protein expression or enzymatic kinetics. Alternatively, protein-protein interaction and, thus, the conservation of interaction sites (e.g. with an intrinsic plastid GGPP synthase isoform) [21] can be involved. This implies that for any other engineered crop plant, the source of PSY might be crucial to achieving high levels of provitamin A production.

Another interesting observation made with GR is that with increasing pathway effectiveness, the proportion of $\beta$-carotene increased relative to the xanthophylls. GR2, for instance, contains as much as $84 \% \beta$-carotene, which is almost double the percentage attained in prototypes. Granted, this direct comparison should not be made because of the different cultivars used, but we have made similar (as yet unpublished) observations with transformed tissue culture cells. The elimination of PSY as a rate-limiting step has revealed that the $\alpha / \beta$ hydroxylases are now the new bottleneck in the pathway. The explanation could lie in the kinetic properties and expression levels of the hydroxylases, but sequestration of $\beta$-carotene must also be considered; reduced accessibility of $\beta$-carotene as a hydroxylase substrate could be the consequence. Carotenoids, when overproduced in chromoplasts, are found sequestered into sites that are different from the plastid membranes in which they were formed. Such sites are for example lipid globules, protein-lipid complexes termed fibrils, or crystals; alternatively the process can be accompanied by plastid membrane 
proliferation [22]. It will be interesting to see whether $\beta$-carotene is found in a different site in GR1 than it is in GR2. Such differences might also impact positively or negatively on bioavailability. A flux-based mechanism of carotenoid deposition, if such a generalization can be made, might provide a means of increasing the relative provitamin A content in tissues, where xanthophylls (lacking provitamin A activity) prevail, such as in maize. Alternatively, downregulation of $\beta$-carotene hydroxylases can be considered if their expression pattern in relevant crop tissues proves to be less complex than shown for leaves of Arabidopsis [13].

Generally, carotenoids degrade over time because of their extended polyene chain, in processes determined by light, temperature, water content of the tissue and by the presence of oxidizing agents [23]. One further possibility is their cleavage by carotenoid oxygenases, a novel class of non-heme-iron enzymes, common in all taxa $[24,25]$. Moreover, certain losses of carotenoids during food processing must be taken into consideration. Investigation of carotenoid storage stability and retention studies are currently underway; these studies will expand as more research materials are generated in field trials. These data, together with the feeding trial mentioned above, should finally provide insight into what is the adequate amount of provitamin A that needs to be produced in GR.

Although GR events have been produced in several Indica, Javanica and Japonica cultivars, the influence of the different biochemical backgrounds of these different genotypes has not yet been studied. The high carotenoid content observed with Cocodrie and Kaybonnet, for instance, could also be the result of the much larger number of events created with these two cultivars, which would adequately account for positional effects impacting on gene expression. Introgression using marker-assisted selection is currently being carried out in Asia, which should provide information to make this distinction. In any case, cross-breeding is required to introgress the GR trait into cultivars of rice, mainly Indica, that are locally adapted in areas with VAD.

\section{Novel approaches - Golden Rice combined with additional nutritional traits}

Given that the amount of provitamin A that accumulates in rice endosperm can be increased depending on the choice of $p s y$ and by event selection, our current view is that the upstream proof-of-concept research for GR-technology has now largely been successfully completed. Equivalent research has just begun for other crops that could assist in combating VAD. However, additional research on GR needs to be conducted to comply with regulatory requirements (Box 2).

A new challenge is to direct research towards combining GR with additional nutritional traits, such as the accumulation of vitamin $\mathrm{E}$, iron and zinc, and to increase the deposition of high-quality protein or essential amino acids in the grain.

Tocopherols and tocotrienols constitute Vitamin E, an essential component of the diet, and have the additional benefit that they help stabilize provitamin A within the food matrix owing to their strong antioxidant properties.
Additional transgenes need to be introduced into GR to increase the vitamin $\mathrm{E}$ content in rice endosperm where it occurs in only small amounts. $\alpha$-Tocopherol is abundant in the rice embryo, but this tissue is removed during milling. There is substantial knowledge of vitamin $\mathrm{E}$ pathway engineering and the full suite of vitamin E pathway genes is available ([26], see the review by Dean DellaPenna in this issue of Trends in Plant Science). Proof-of-concept experiments are required to determine the optimal gene complement for rice endosperm and if and how provitamin $\mathrm{A}$ and vitamin $\mathrm{E}$ production can be increased simultaneously because both pathways share a common precursor molecule (GGPP; Figure 2).

We need to increase our knowledge of iron metabolism in plants to be able to deal with the accumulation of iron and zinc, for which micronutrient deficiencies are most severe. A third of the world's population is estimated to be chronically anemic because of iron deficiency (http:// www.who.int/nut/ida.htm). Transgenic approaches to increase the iron content have been carried out in the past but the changes were minor. This is because the primary determinant of seed mineral content is the loading of metals into the phloem transport pathway [27]. Forty-three genes encoding proteins from five families are currently being considered as candidates to be involved in iron-homeostasis in rice [28]. Such multi-gene traits are difficult to assess using GMO technologies and require extensive proof-of-concept experimentation to determine the rate-limiting steps in iron accumulation in the endosperm. In parallel, plant breeders have begun screening germplasm collections for variation in mineral content. Thus far, this has resulted in finding a fourfold variation for both seed $\mathrm{Fe}$ and $\mathrm{Zn}$ [29]. Thus, one strategy could be to use high-Fe and - Zn rice germplasm for introgression of the GR trait. In addition, the reduction in the content of anti-nutritive factors for bivalent metal cations and the increase of promoters of bioavailability (the capability to absorb the ingested iron or zinc) are under investigation. An open approach to determine the biochemical basis of iron bioavailability is currently underway, based on the identification of QTLs.

The increase of protein complement in GR or the achievement of a balanced amino acid composition are further challenges. Milled rice is low in protein concentration $(7 \%)$ but, compared with other cereals, the protein quality is relatively good in terms of amino acid composition. This is attributed to a relatively low proportion of prolamin (negligible or devoid of lysine) as storage protein, whereas the glutelins predominate. Nevertheless, lysine is limiting in rice, followed by threonine (S.K. Vasal, http://www.fao.org/documents/ show_cdr.asp?url_file =/docrep/007/y5019e/y5019e0b. $\mathrm{htm})$. So far, the protein content and quality of rice has not been improved using conventional breeding and mutant screening approaches.

Although a GMO approach is straightforward in theory, past experience has shown that there is often a trade-off in cereals, where enhanced protein levels can lead to 
diminished yields or to alterations in other nutritional or grain quality components [30].

Changing the content of free essential amino acids by engineering their respective biosynthetic enzymes is an alternative option that has received considerable attention in the past. However, feedback-regulatory phenomena, increased catabolic activities and aberrant phenotypes have often been observed [30].

Given that the protein supply in human nutrition increasingly relies on plant sources, and the importance of rice in this respect, which feeds $\sim 50 \%$ of the world's population, it remains desirable to direct major efforts into proof-of-concept experimentation along both strategies, until a viable solution is found.

\section{Concluding remarks}

The development of GR has progressed significantly during recent years and most of the current efforts are directed towards making it available for farmers and consumers in developing countries and, thus, to begin contributing towards combating VAD.

The continued development of GR to meet the challenge of providing a provitamin A biofortified rice and the complement of micronutrients necessary to combat additional micronutrient deficiency disorders will require the close collaboration of international research consortia willing to amalgamate their complementary expertise and knowledge in biochemistry, transformation technologies, breeding, deregulation, nutrition and end-user outreach activities. Most importantly, National Agricultural Research Institutions in countries with VAD need to be involved early on in the process, not only because of their complementary knowledge but also to create national ownership of the project. Two consortia have recently been formed. One is HarvestPlus (http://www.harvestplus.org), which targets the nutritional improvement of a wide variety of crop plants, mostly through breeding. The other is in the Grand Challenges in Global Health initiative (http://www.gcgh.org/subcontent.aspx?SecID = 390), which is funded by the Bill and Melinda Gates Foundation, targeting banana, cassava, sorghum and rice, mostly through genetic modification. Such consortia are expected to continue to develop into focal points for a novel type of 'Green Revolution' in which the well-known issues of food nutritional content, which had been neglected in the past, will be addressed.

Given that most of these efforts are currently in the public sector, some structural change and capacity building seem inevitable. Both research and development need to be treated equally in terms of recognition and funding. Public sector scientists are rewarded by scientific publication as the only measure of professional excellence. The number and cutting-edge quality of publications are decisive for driving a successful career; the focus is on scientific novelty. Moreover, current funding sources are governed by reviewing scientists, mostly raised in that same tradition. Consequently, scientists in the public sector, particularly younger scientists, will be reluctant to sacrifice their productive years with product development research that involves a great deal of repetitious work lacking novelty. Therefore, most public sector scientists, after an initial discovery, might not pursue the topic towards application, leaving this feat to 'other scientists' (http://www.economia.uniroma2.it/conferenze/icabr2003/ papers/Developing_Coutries/Dubock.zip; http://www. economia.uniroma2.it/conferenze/icabr2005/papers/

Dubock_paper.pdf).

In the past, these 'other scientists' have mainly worked for R\&D-based companies. Product development requires focusing on the product objective, and combining all necessary resources to achieve that goal. A completely different set of logistics, focus, skills and specific expertise has been established that is hardly found in public sector research institutions. Private sector scientists are being rewarded by their employers for conducting product development; this does not require (but does not exclude) publication. Such capacity needs to be built into some selected public research institutions. As a time-saving and eventually less costly alternative, a novel type of publicprivate partnership should be considered, in which the public sector pays a company for contributing the elements of the project for which they have proven expertise and excellence. In particular, there are products of high general interest or demand such as GR that are not commercially interesting. Hence, the public sector should take over the responsibility of generating and delivering such products but, at the same time, should benefit from and integrate the expertise that the private sector has to offer.

\section{Acknowledgements}

We thank Gerard Barry, Camilla Beech, Rachel Drake, Adrian Dubock, Stella Dubock, Jorge Mayer and Ingo Potrykus for discussion on the manuscript.

\section{References}

1 Ye, X. et al. (2000) Engineering the provitamin A ( $\beta$-carotene) biosynthetic pathway into (carotenoid-free) rice endosperm. Science 287, 303-305

2 Burkhardt, P.K. et al. (1997) Transgenic rice (Oryza sativa) endosperm expressing daffodil (Narcissus pseudonarcissus) phytoene synthase accumulates phytoene, a key intermediate of provitamin A biosynthesis. Plant J. 11, 1071-1078

3 Lucca, P. et al. (2001) Effective selection and regeneration of transgenic rice plants with mannose as selective agent. Mol. Breed. 7, 43-49

4 Hoa, T.T.C. et al. (2003) Golden Indica and Japonica rice lines amenable to deregulation. Plant Physiol. 133, 161-169

5 Datta, K. et al. (2003) Bioengineered 'golden' Indica rice cultivars with $\beta$-carotene metabolism in the endosperm with hygromycin and mannose selection systems. Plant Biotechnol. J. 1, 81-85

6 Zimmermann, R. and Qaim, M. (2004) Potential health benefits of Golden Rice: a Philippines case study. Food Policy 29, 147-168

7 Lois, L.M. et al. (2000) Carotenoid biosynthesis during tomato fruit development: regulatory role of 1-deoxy-D-xylulose 5-phosphate synthase. Plant J. 22, 503-513

8 Paine, J.A. et al. (2005) Improving the nutritional value of Golden Rice through increased pro-vitamin A content. Nat. Biotechnol. 23, 482-487

9 Shewmaker, C.K. et al. (1999) Seed-specific overexpression of phytoene synthase: increase in carotenoids and other metabolic effects. Plant J. 20, 401-412

10 Giuliano, G. et al. (2003) Carotenoid oxygenases: cleave it or leave it. Trends Plant Sci. 8, 145-149

11 West, C.E. et al. (2002) Consequences of revised estimates of carotenoid bioefficacy for dietary control of vitamin A deficiency in 
developing countries. Supplement: Proceedings of the XX International Vitamin A Consultative Group Meeting. J. Nutr. 132, 2920S-2926S (http://www.nutrition.org/cgi/content/full/132/9/2920S)

12 U.S. Institute of Medicine, Food and Nutrition Board, Standing Committee on the Scientific Evaluation of Dietary Reference Intakes (2000) Dietary Reference Intakes for Vitamin A, Vitamin K, Arsenic, Boron, Chromium, Copper, Iodine, Iron, Manganese, Molybdenum, Nickel, Silicon, Vanadium, and Zinc 2000, National Academy Press, Washington, DC, USA

13 Tian, L. and DellaPenna, D. (2004) Progress in understanding the origin and functions of carotenoid hydroxylases in plants. Arch. Biochem. Biophys. 430, 22-29

14 Romer, S. et al. (2000) Elevation of the provitamin A content of transgenic tomato plants. Nat. Biotechnol. 18, 666-669

15 Bartley, G.E. et al. (1999) Two Arabidopsis thaliana carotene desaturases, phytoene desaturase and zeta-carotene desaturase, expressed in Escherichia coli, catalyze a poly-cis pathway to yield pro-lycopene. Eur. J. Biochem. 259, 396-403

16 Isaacson, T. et al. (2002) Cloning of tangerine from tomato reveals a carotenoid isomerase essential for the production of beta-carotene and xanthophylls in plants. Plant Cell 14, 333-342

17 Park, H. et al. (2002) Identification of the carotenoid isomerase provides insight into carotenoid biosynthesis, prolamellar body formation, and photomorphogenesis. Plant Cell 14, 321-332

18 Al-Babili, S. et al. (1999) CPTA modulates levels of carotenogenic proteins and their mRNAs and affects carotenoid and ABA content as well as chromoplast structure in Narcissus pseudonarcissus flowers. Plant Biol. 1, 607-612

19 Schaub, P. et al. (2005) Why is golden rice golden (yellow) instead of red? Plant Physiol. 138, 441-450

20 Carol, P. and Kuntz, M. (2001) A plastid terminal oxidase comes to light: implications for carotenoid biosynthesis and chlororespiration. Trends Plant Sci. 6, 31-36

21 Okada, K. et al. (2000) Five geranylgeranyl diphosphate synthases expressed in different organs are localized into three subcellular compartments in Arabidopsis. Plant Physiol. 122, 1045-1056
22 Camara, B. et al. (1995) Biochemistry and molecular biology of chromoplast development. Int. Rev. Cytol. 163, 175-247

23 Bonnie, T.Y.P. and Choo, Y.M. (1999) Oxidation and thermal degradation of carotenoids. J. Palm Oil Res. 1, 62-78

24 Bouvier, F. et al. (2005) Oxidative tailoring of carotenoids: a prospect towards novel functions in plants. Trends Plant Sci. 10, 187-194

25 Kloer, D.P. et al. (2005) The structure of a retinal-forming carotenoid oxygenase. Science $308,267-269$

26 Ajjawi, I. and Shintani, D. (2004) Engineered plants with elevated vitamin E: a nutraceutical success story. Trends Biotechnol. 22, 104-107

27 Grusak, M.A. (2002) Enhancing mineral content in plant food products. J. Am. Coll. Nutr. 21, 178S-183S

28 Gross, J. et al. (2003) Iron homeostasis related genes in rice. Genet. Mol. Biol. 26, 4, 477-497

29 Gregorio, G.B. et al. (2000) Breeding for trace mineral density in rice. Food Nutr. Bull. 21, 382-386

30 Sun, S.S.M. et al. (2003) Transgenic approaches to improve the nutritional quality of plant proteins. In Vitro Cell. Dev. Biol. Plant 40, 155-162

31 Bouis, H. (2000) Commercial vegetable and polyculture fish production in Bangladesh: their impacts on household income and dietary quality. Food and Nutrition Bulletin Special Issue 21, 482-487

32 Sommer, A. and Davidson, F.R. (2002) Assessment and control of vitamin A deficiency: the Annecy Accords. J. Nutr. 132, $2845 \mathrm{~S}-2850 \mathrm{~S}$

33 Kuiper, H.A. et al. (2001) Assessment of the food safety issues related to genetically modified foods. Plant J. 27, 503-528

34 Rodriguez-Concepcion, M. and Boronat, A. (2002) Elucidation of the methylerythritol phosphate pathway for isoprenoid biosynthesis in bacteria and plastids. A metabolic milestone achieved through genomics. Plant Physiol. 130, 1079-1089

35 Isaacson, T. et al. (2004) Analysis in vitro of the enzyme CRTISO establishes a poly-cis-carotenoid biosynthesis pathway in plants. Plant Physiol. 136, 4246-4255

\title{
Plant Biology 2006
}

(Annual meeting American Society of Plant Biologists)

\author{
05-09 August 2006 \\ Boston, MA, USA
}

For more information see http://www.aspb.org/meetings/

ISPMB 2006

8th International Congress of Plant Molecular Biology

20-25 August 2006

Adelaide Convention Centre, Australia

For more information see http://www.sallyjayconferences.com.au/ispmb2006/

Enquiries: Sally Jay - ispmb@sallyjayconferences.com.au 